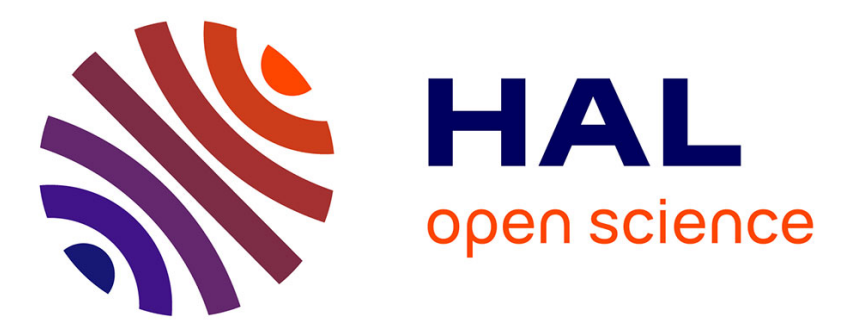

\title{
Accuracy of a New Robotically Assisted Technique for Total Knee Arthroplasty: A Cadaveric Study
}

Sebastien Parratte, Andrew J. Price, Lee M. Jeys, William F. Jackson, Henry

D. Clarke

\section{- To cite this version:}

Sebastien Parratte, Andrew J. Price, Lee M. Jeys, William F. Jackson, Henry D. Clarke. Accuracy of a New Robotically Assisted Technique for Total Knee Arthroplasty: A Cadaveric Study. The Journal of Arthroplasty, 2019, 34 (11), pp.2799-2803. 10.1016/j.arth.2019.06.040 hal-02528233

\section{HAL Id: hal-02528233 \\ https://hal.science/hal-02528233}

Submitted on 13 Apr 2020

HAL is a multi-disciplinary open access archive for the deposit and dissemination of scientific research documents, whether they are published or not. The documents may come from teaching and research institutions in France or abroad, or from public or private research centers.
L'archive ouverte pluridisciplinaire HAL, est destinée au dépôt et à la diffusion de documents scientifiques de niveau recherche, publiés ou non, émanant des établissements d'enseignement et de recherche français ou étrangers, des laboratoires publics ou privés. 


\title{
Accuracy of a New Robotically Assisted Technique for Total Knee Arthroplasty: A Cadaveric Study
}

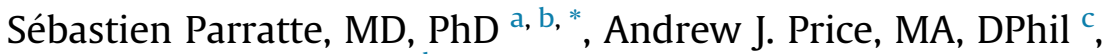 \\ Lee M. Jeys, MSc, FRCS ${ }^{\mathrm{d}}$, William F. Jackson, FRCS ${ }^{\mathrm{c}}$, Henry D. Clarke, MD ${ }^{\mathrm{e}}$ \\ ${ }^{a}$ Adult Reconstructive Surgery, International Knee and Joint Centre, Abu Dhabi, United Arab Emirates \\ ${ }^{\mathrm{b}}$ Adult Reconstructive Surgery, Institute for Locomotion, Aix-Marseille University, Marseille, France \\ c Adult Reconstructive Surgery, Nuffield Orthopaedic Centre, Oxford, United Kingdom \\ ${ }^{\mathrm{d}}$ Adult Reconstructive Surgery, Royal Orthopaedic Hospital, Birmingham, United Kingdom \\ e Adult Reconstructive Surgery, Mayo Clinic, Phoenix, AZ
}

Keywords:
TKA
robotic surgery
accuracy
computer-assisted surgery
in vitro

\begin{abstract}
A B S T R A C T
Background: Although the utility of robotic surgery has already been proven in cadaveric studies, it is our hypothesis that this newly designed robotically assisted system will achieve a high level of accuracy for bone resection. Therefore, we aimed to analyze in a cadaveric study the accuracy to achieve targeted angles and resection thickness.

Methods: For this study, 15 frozen cadaveric specimens (30 knees) were used. In this study, Zimmer Biomet (Warsaw, IN) knees, navigation system, and robot (ROSA Knee System; Zimmer Biomet) were used. Eight trained, board-certified orthopedic surgeons performed robotically assisted total knee arthroplasty implantation using the same robotic protocol with 3 different implant designs. The target angles obtained from the intraoperative planning were then compared to the angles of the bone cuts performed using the robotic system and measured with the computer-assisted system considered to be the gold standard. For each bone cut the resection thickness was measured 3 times by 2 different observers and compared to the values for the planned resections.

Results: All angle mean differences were below $1^{\circ}$ and standard deviations below $1^{\circ}$. For all 6 angles, the mean differences between the target angle and the measured values were not significantly different from 0 except for the femoral flexion angle which had a mean difference of $0.95^{\circ}$. The mean hip-knee-ankle axis difference was $-0.03^{\circ} \pm 0.87^{\circ}$. All resection mean differences were below $0.7 \mathrm{~mm}$ and standard deviations below $1.1 \mathrm{~mm}$.

Conclusion: Despite the fact that this study was funded by Zimmer Biomet and only used Zimmer Biomet implants, robot, and navigation tools, the results of our in vitro study demonstrated that surgeons using this new surgical robot in total knee arthroplasty can perform highly accurate bone cuts to achieve the planned angles and resection thickness as measured using conventional navigation.
\end{abstract}

The analysis of the total knee arthroplasty (TKA) demonstrates that up to $20 \%$ of the patients are not satisfied with their knee function after TKA [1]. Pain, instability, and limitation of the range of

\footnotetext{
Funding: This study is funded Zimmer Biomet.

One or more of the authors of this paper have disclosed potential or pertinent conflicts of interest, which may include receipt of payment, either direct or indirect institutional support, or association with an entity in the biomedical field which may be perceived to have potential conflict of interest with this work. For full disclosure statements refer to https://doi.org/10.1016/j.arth.2019.06.040.

* Reprint requests: Sébastien Parratte, MD, PhD, International Knee and Joint Centre, Abu Dhabi, United Arab Emirates.
}

motion are the most commonly reported symptoms among unsatisfied patients [1,2]. Inadequate alignment and gap balancing have been implicated as major potential causes of these limitations [2,3]. During the last decade, the philosophy of restoring a neutral mechanical axis to the surgical limb, associated with performing extensive soft tissue releases, has been questioned [4-6]. Although most surgeons are still aiming for restoration of strict neutral limb alignment, alternative concepts of alignment have been proposed, such as preservation of a more anatomical alignment [4-6]. These techniques are purportedly associated with less need to perform extensive ligament releases [4-6]. As standard mechanical instrumentation might not be accurate enough to achieve either of these, 
perfect neutral alignment or specific anatomic alignment, tools such as computer-assisted surgery, patient-specific instrumentation (PSI), and more recently robotic-assisted TKA have been introduced [7]. Different robot systems for performing TKA are now available [7]. They require preoperative computed tomography (CT) scans and incorporate different end effectors from burrs to haptic saws, with potential advantages and drawbacks for each technology [7]. A different and recently introduced robotic-arm system that incorporates robotic placement of the cutting jigs and dynamic ligament balance evaluation has been developed. This system can be considered as a collaborative robotic system where the robot is holding and placing the cutting jig, while the surgeon is in full control of sawing through the jig. This system is different compared to the other systems available on the market such as the haptic arm keeping the surgeons in limited boundaries. This system is also different from the milling robotic systems available on the market, either based on a passive mode (ie, the robot is not doing the action but the surgeon is actively involved in the surgical process holding the robotic milling device that is retracting when reaching the boundaries) or based on an active mode robotic milling type of device where the surgeon is not involved at all during the milling process, the robot being active and doing the milling process [7]. The guiding principles of this system are simplicity; preservation of the natural surgical flow; minimization of extra-time related to robotic surgery as observed in alternative systems; and achieving high levels of accuracy for specific angular and resection thickness targets. This study was designed to simulate the in vivo capabilities of this new robotic system and analyze the accuracy of this system to perform planned bone cuts. The goal of this study is to determine the accuracy of the bone cuts made using this newly designed robotically assisted robotic system for TKA. Therefore, we analyzed in a cadaveric study the accuracy relative to target angles for the following measures: hip-knee-ankle axis (HKA), tibial cut (frontal and sagittal), femoral cut (frontal and sagittal) as measured using a validated computer-assisted navigation system. We also measured the accuracy relative to target resection thickness values for the following bone cuts: tibial resection (medial and lateral tibial plateaus), and distal and posterior femoral condylar resections, as measured using a validated caliper.

\section{Materials and Methods}

For this study, 15 frozen cadaveric specimens (30 knees) were used. The mean age at the time of death was $78.7 \pm 11.1$ years with a mean body mass index of $22.7 \mathrm{~kg} / \mathrm{m}^{2}$. Nine of the 15 cadavers were females. Eight trained, board-certified orthopedic surgeons were involved in the cadaveric evaluation. All the surgeons involved in the study were knee arthroplasty specialists with at least 5 years of practice and performing more than 100 knee arthroplasties per year. All the surgeons were familiar with the knee system used in the study. However, as the experience of these surgeons using smart tools was different, all the surgeons involved in the study received the same standardized training for the ROSA Knee platform. This training included a theoretical training on the features of the system, the practice of what they learned on 3 TKAs performed on sawbones and 2 TKAs performed on cadavers before starting the study. For the study, each of them performed either 4 knees (for 6 surgeons) or 3 knees (for 2 surgeons). Three different implant designs (Persona, Zimmer Biomet, Warsaw, IN; NexGen, Zimmer Biomet; Vanguard, Zimmer Biomet) were used to avoid any implant selection bias. For each knee, the same robotic protocol was used (ROSA Knee System; Zimmer Biomet). Using the system's imageless mode, coordinate systems were generated from landmarks taken on the femur and tibia after arthrotomy. An intraoperative plan was determined, and bone resections were then performed. As described later in the manuscript, to standardize the protocol, the intraoperative plan aimed to restore the neutral mechanical axis (tibia $90^{\circ}$ and femur $90^{\circ}$ ) for all the knees and resections were performed aiming for a $10-\mathrm{mm}$ polyethylene. The target angles obtained from the intraoperative plan were then measured and compared to the bone cuts performed with the robotic system as measured with a validated computer-assisted navigation system (ORTHOsoft; Zimmer Biomet) considered to be the gold standard as previously published $[8,9]$. For each bone cut the resection thickness was measured using a caliper 3 times by 2 different observers and compared to the values given to the planned resections [10].

\section{Robotic Procedure}

For each cadaveric knee, 2 rigid body trackers (one on the femur and one on the tibia) were implanted. The robot was calibrated. The registration of the femoral and tibial landmarks was then performed. Following this step, intraoperative planning was performed by each surgeon, using the dedicated software, to determine the ideal resection thickness and angle to obtain a balanced and well-aligned TKA, following the basic principles of TKA [2] (Fig. 1). To standardize the procedure the target HKA was $180^{\circ}$, with $90^{\circ}$ for both the tibia and femoral coronal angles. The targeted femoral flexion angle (sagittal position of the femoral implant defined as the angle between the implant and the line crossing the center of the knee and the center of the hip in a 3-dimensional coordinate system) was $3^{\circ}$ and the tibial slope was adapted to the type of implant (from $3^{\circ}$ to $7^{\circ}$ ). All the planned values for both the angles and the resection thickness were recorded. ROSA Knee was then used to perform the distal femoral cut first immediately followed by the tibial cut. The principle of ROSA Knee surgery is simple: the robotic arm positions and holds the universal cutting jig in the desired location as determined by the surgical plan. Once the cutting jig is set in the correct position it is pinned to the bone and the surgeon then performs the cuts (Fig. 2). Once the distal femoral and proximal tibial cuts were performed, the robotic arm returned to its resting position and the extension space was evaluated and final planning was optimized. The robotic arm was then used to position the drilling holes for the dedicated classic 4-in-1 anterior-posterior cutting jig that sets the anterior-posterior position and rotation of the femoral component. Next, the 4 remaining femoral cuts were performed and the flexion space was evaluated. Final steps of the procedure were then performed using standard instrumentation for the preparation of the tibial metaphysis and the patella. Trial implants were then inserted, and the mechanical axis of the leg was recorded for comparison with the operative plan.

\section{Navigation and Angle Measurement}

In this study, cuts were made with ROSA Knee and values were measured with the ORTHOsoft Total Knee Navigation system (Zimmer Biomet) [8,9]. The average accuracy of this infrared camera-based imageless navigation system has been reported to be within $1 \mathrm{~mm}$ for the resection thickness and $0.4^{\circ}$ for angle measurement $[8,9]$. The same rigid bodies were used for both the ROSA Knee procedure and the navigation measurements. For the navigation measurements, the usual registration process was performed immediately after the bone registration procedure for ROSA Knee was completed. For each bone cut, a handheld validation tool was placed on the cuts in order to verify and record alignment in the sagittal and coronal planes. This tool is dedicated to the measurement of each of the bone cuts (particularly the distal femoral resection and the tibial cut) made of a flat surface using a navigation tracker and handled by the surgeon who is applying the flat surface of the tool onto the flat surface of the cut made. This tool has been validated in previous studies $[8,9]$. The 


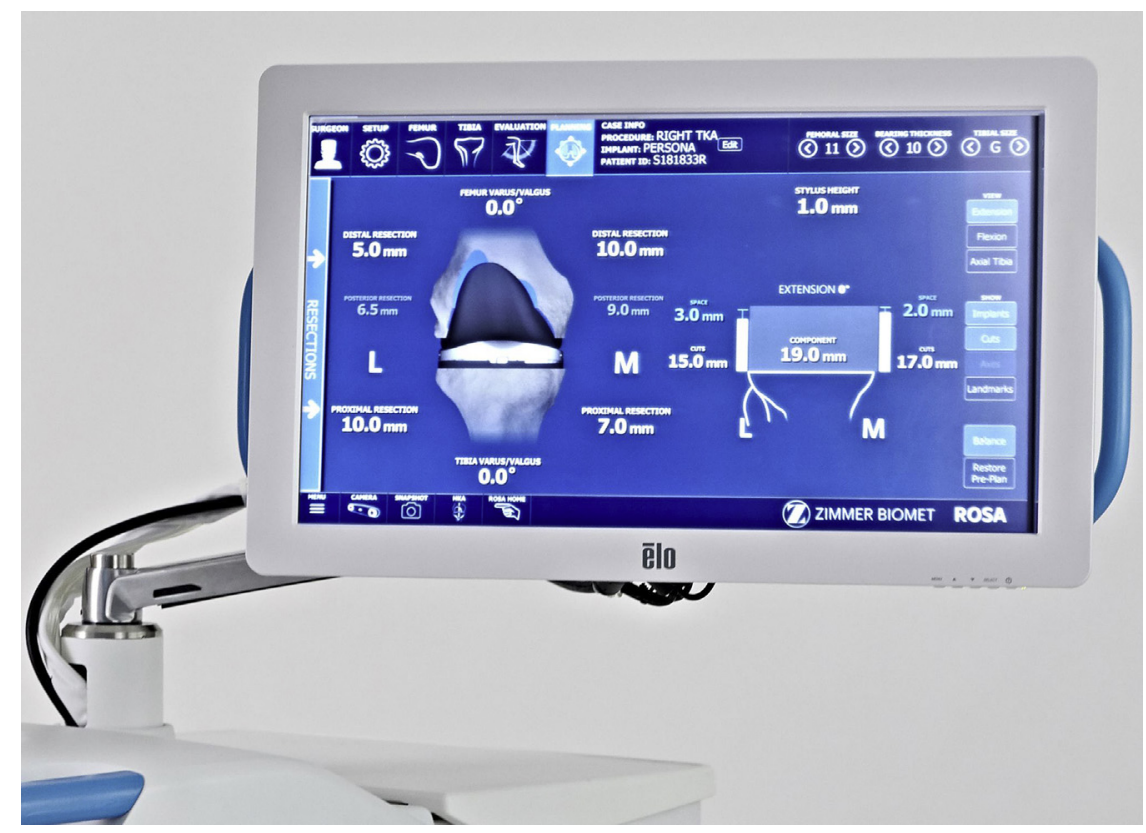

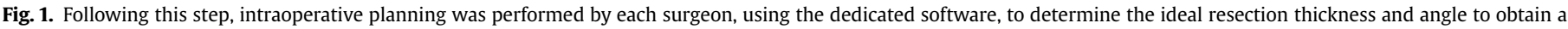
balanced and well-aligned TKA.

computer system then calculated the discrepancy between the planned cut (depth, frontal, and sagittal angles) compared to the cut performed using the robotic system.

\section{Measurement of Resection Thickness}

After performing each cut using ROSA Knee, the thickness of the resected bone was measured using a dedicated caliper [10]. Each

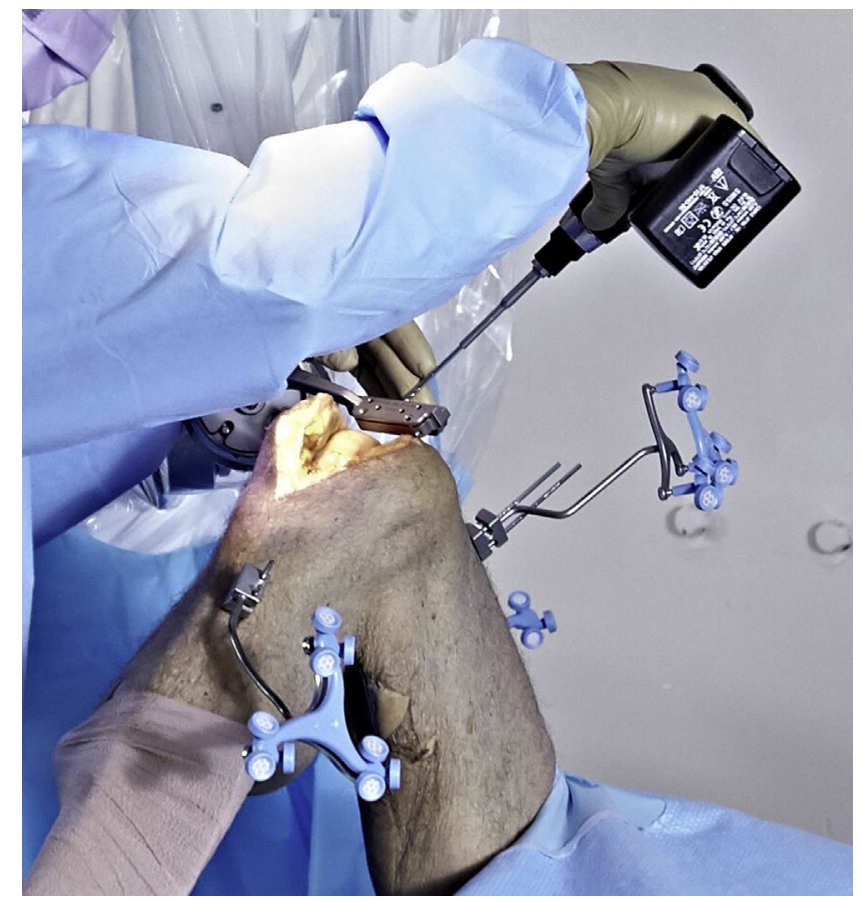

Fig. 2. The principle of ROSA Knee surgery is simple: the Robotic arm positions and holds the universal cutting jig in the desired location as determined by the surgical plan. Once the cutting jig is set in the correct position it is pinned to the bone and the surgeon then performs the cuts. cut was measured 3 times by 2 different independent observers. For each cadaver, the thickness of the following cuts was measured: medial and lateral tibial plateau, medial and distal condyles, posterolateral and posteromedial cuts of the femur.

\section{Statistical Analysis}

To confirm the reliability of the measurements, interobserver and intraobserver comparisons of resection measurements at 2 different time points were performed with use of the method of Bland and Altman on 40 randomly chosen samples from the study [11]. For these 40 samples, the intraobserver and interobserver measurement errors were $<0.5 \mathrm{~mm}$ for all of the analyzed resections. The values measured with ROSA Knee and with the navigation system were compared to the target values recorded in the surgical plan to evaluate the capacity to execute the plan using a previously described method [8,9]. After checking for data normality, descriptive statistics (mean, standard deviation, and range) were calculated. Dedicated $t$-test for paired samples was used to compare the difference between the target values and the measured values considering the navigation system as the gold standard. The proportion of differences within $\pm 1^{\circ}$ and $\pm 2^{\circ}$ was calculated for the alignment values. Similarly, the proportion of differences within $\pm 1 \mathrm{~mm}$ and $\pm 2 \mathrm{~mm}$ was calculated for the resection thicknesses. Prediction intervals for a single future measurement were also calculated to identify the range of values where the planned values and the measured values would likely fall within. Confidence intervals of $95 \%$ were set a priori for both the $t$ test and the $99 \%$ prediction interval. Statistical significance was set at $P<.05$ for all statistical tests. All statistics were performed using SPSS software version 21 (SPSS Inc., Chicago, IL).

\section{Results}

For all 30 specimens, the differences between the target angles and measured angles (computed as described above) were shown to follow a normal distribution, of sufficient sample size and are reproduced on Table 1 . In all cases, the mean differences are below 
Table 1

Difference Between the Targeted Angles and the Angles Measured With the Optical Navigation System (ORTHOsoft, Zimmer Biomet, IN).

\begin{tabular}{|c|c|c|c|c|c|c|c|c|}
\hline Angles & Mean \pm SD & RMS & $P$ Value & Range $\left(^{\circ}\right)$ & $\%$ Within $3^{\circ}$ & $\%$ Within $2^{\circ}$ & $\%$ Within $1^{\circ}$ & 99\% PI \\
\hline \multicolumn{9}{|l|}{ Femoral } \\
\hline Coronal & $0.03 \pm 0.51$ & 0.5 & .71 & $-1.1,1.5$ & 100 & 100 & 93 & $-1.7,1.7$ \\
\hline Sagittal & $-0.95 \pm 0.88$ & 1.29 & $<.00$ & $-3.4,0.6$ & 97 & 87 & 63 & $-3.9,2.0$ \\
\hline \multicolumn{9}{|l|}{ Tibial } \\
\hline Coronal & $-0.06 \pm 0.69$ & 0.68 & .62 & $-1.1,1.7$ & 100 & 100 & 87 & $-2.4,2.2$ \\
\hline Slope & $0.20 \pm 0.84$ & 0.84 & .2 & $-1.3,1.7$ & 100 & 100 & 73 & $-2.6,3.0$ \\
\hline \multicolumn{9}{|l|}{ Global } \\
\hline HKA & $-0.03 \pm 0.87$ & 0.85 & .86 & $-2.3,1.6$ & 100 & 97 & 73 & $-2.9,2.9$ \\
\hline
\end{tabular}

SD, standard deviation; HKA, hip-knee-ankle angle; RMS, root mean square; PI, predictive intervals.

$1^{\circ}$ and the standard deviation is less than $1^{\circ}$. Except for the femoral sagittal angle difference, the mean differences between the planned angles and the measured values were close to 0 and not significantly different from 0 . The femoral sagittal angle mean difference was $-0.95^{\circ}$ (Table 1 ).

For each 30 specimens, 6 different resection measurements were taken and the differences between the target resection and the measured resection were computed as described above. The measures were shown to follow a normal distribution, of sufficient sample size and are reproduced on Table 2 . In all cases, the mean difference was below $1 \mathrm{~mm}$ and the standard deviation was less than $1.1 \mathrm{~mm}$. For all but 2 measurements, the mean difference between the planned and measured resection thickness was not significantly different from 0 . A statistically significant mean difference of $0.3 \mathrm{~mm}$ was observed for the distal femoral condyle and $0.66 \mathrm{~mm}$ for the medial tibial plateau. (Table 2). In all cases, the standard deviation was below $1 \mathrm{~mm}$.

\section{Discussion}

A different and recently introduced robotic system based on the use of a robotic-arm to assist the surgeon during TKA to perform bone cuts has been developed. This system can be considered as a collaborative robotic system (cobot) where the robot is positioning and holding the cutting jigs, while the surgeon performs the cuts using a conventional saw. It is our hypothesis for this study that this newly designed robotically assisted system would achieve a high level of accuracy (within a mean error of $1^{\circ}$ for angular values and within a mean error of $1 \mathrm{~mm}$ for resection thickness values). The results of our study demonstrated that the cuts performed using ROSA Knee were very accurate relative to plan. Regarding the resection angles there were no significant differences observed between the planned and the measured values, except for femoral flexion that had a mean difference of $-0.95^{\circ}$. For the resection thickness values, no differences were observed except for the distal medial femoral and medial proximal tibial cuts. The HKA mean difference was computed at $-0.03^{\circ} \pm 0.87^{\circ}$.

Several limitations in our study should be outlined: First, this study is supported by Zimmer Biomet and exclusively used Zimmer
Biomet implants and navigation system for the testing of the accuracy. Only Zimmer Biomet implants were used as the robotic platform is not an open platform, rather it was designed for use only with Zimmer Biomet implants. The navigation system used for the validation of the angles has been validated in the past and the methodology used in previous study for the validation of smart tools [8-10]. Second, we did not complete the cementation of the implant and we did not perform any final 3-dimensional evaluation using CT scan. Our study was however designed to evaluate the accuracy of the cuts compared to plan. To remove the potential error related to the cementation, we decided to follow a previously published method (based on navigation and direct caliper measurement) [8-10]. This method offers the advantage of measuring directly the values of the cuts and the resection thickness [8-10]. The third limitation of our study is related to the absence of measurement of the rotational alignment of the implants. Limited accuracy for the navigation systems concerning the rotation measurement has been described in the literature with up to $6^{\circ}$ of error related to interindividual and intraindividual variations during the palpation of the epicondyles and analysis of the rotation would have required a different study design potentially less adapted to the validation of our hypothesis focusing on the accuracy of bony resection [12]. In fact, we would have needed a CT scan to validate the rotation after implantation of the final implants. That would have introduced a potential bias related to either the impaction of the implant for a cementless implant or the quality of the cementation for a cemented implant. Therefore, we limited our goal to one objective only: the accuracy of the bone cut. Despite this limitation, the accuracy of the resection of the posterior condyles was measured and the accuracy confirmed which mean that once properly defined and planned, the robot will help us to reach the targeted rotation.

The fourth limitation is related to the in vitro design of the study. The cadavers had no known significant osteoarthritis and errors are less likely with normally aligned cadavers, as opposed to the deformed legs of clinical cases where the bone quality was very poor and the ligament tension was hardly comparable to an in vivo situation. Statistically, if larger numbers of cadavers were done, it is

Table 2

Difference Between Planned Bone Resections and Bone Resections Measured With the Caliper

\begin{tabular}{|c|c|c|c|c|c|c|}
\hline Parameters & Mean \pm SD & $P$ Value & Range (mm) & \% Within $2 \mathrm{~mm}$ & $\%$ Within $1 \mathrm{~mm}$ & 99\% PI \\
\hline \multicolumn{7}{|l|}{ Femoral } \\
\hline Medial distal & $0.35 \pm 0.84$ & .03 & $-1.8,2.5$ & 97 & 80 & $-2.5,3.2$ \\
\hline Lateral distal & $0.06 \pm 0.86$ & .71 & $-1.9,2.5$ & 97 & 83 & $-2.8,2.9$ \\
\hline Medial posterior & $0.23 \pm 0.93$ & .21 & $-1.6,2.4$ & 93 & 75 & $-2.9,3.4$ \\
\hline Lateral posterior & $-0.05 \pm 0.76$ & .71 & $-1.9,1.3$ & 100 & 82 & $-2.6,2.5$ \\
\hline \multicolumn{7}{|l|}{ Tibial } \\
\hline Medial plateau & $0.66 \pm 0.64$ & $<.00$ & $-0.6,1.7$ & 100 & 63 & $-1.5,2.8$ \\
\hline Lateral plateau & $0.15 \pm 1.08$ & .46 & $-3.4,1.8$ & 93 & 73 & $-3.5,3.8$ \\
\hline
\end{tabular}

SD, standard deviation; PI, prediction interval. 
likely that some would be found with errors. A fifth limitation comes from our measuring equipment. Using today's gold standard in navigation technology, our measurement resolution depends on the documented performance of the optical navigation system, as mentioned earlier, with an angular accuracy of $0.4^{\circ}$ and a size accuracy of $1 \mathrm{~mm}[8,9]$. As seen in both tables, our mean differences correlate well with the accuracy of our measurement tool. Last but not the least, the ligament laxity was not assessed as the goal of the study was only to analyze the accuracy of the robot to achieve the planned bone cut resection targets. Despite these limitations, this study can be considered as a first step in the validation process of this system and in the demonstration of its accuracy.

To date, there are limited published studies evaluating the results of the accuracy of robotically assisted TKA. The first study reported results of a robotically assisted TKA using a navigated burr [13]. Two techniques were used in this prior study: for 4 of 18 cadavers, the burr was used for the entire procedure and all the bone cuts were performed with the burr; for the remaining 14 cadavers, the burr was used to drill holes for the jigs, the jigs were applied, and then the cuts were performed like in a conventional technique using a standard saw [13]. The results showed that errors ranged from 0.6 to 2.4 $\mathrm{mm}$ or degrees for the femoral and tibial implants, with the higher errors observed for femoral flexion [13]. This higher risk of error for the femoral flexion was also observed in our study. The authors did not report the root mean square errors nor any prediction intervals; direct comparisons with our study are therefore limited [13]. Furthermore, the number of knees used was not sufficient to reach any level of significance [13]. Another publication reported a cadaveric study comparing TKA implanted on cadavers with either a robotic-arm technique or a conventional instrument system [14]. In that comparative study, a single surgeon performed robotic-assisted or conventional instrument TKA on one leg each of 6 cadavers, for a total of 6 of each type [14]. A variety of femoral and tibial angular measures generally revealed significantly reduced bone cutting errors for the robotic-assisted group [14]. Unfortunately, no measures of planned vs achieved resection thicknesses were provided in that paper [14]. Study design and reporting differences make direct comparisons to the current work difficult, however, bone cut error variances in that report appear generally consistent with our current findings [14]. An analysis of the resections was not directly performed [14]. Rather, the authors evaluated the accuracy of the robotic technique by comparing the position of the trials relative to the plan [14]. Consequently, the different study design limits a direct comparison with our study [14].

The analysis of the amount of resection has however been performed in previous navigation studies [10] and PSI studies [8,9]. The results of our study demonstrate that higher levels of accuracy can be achieved with robotic surgery compared to navigation $[8-10,15,16]$. The differences observed might be explained by the fact that the robot, while optimizing the jig placement according to the surgical plan, is following any potential movement of the leg during the optimal placement of the jig. The jig is then pinned to the bone by the surgeon and the robot is locked in place. This sequence is creating an extremely solid construct with no micro-mobility which opposes to conventional navigation, where the surgeon has to hold the jigs, trying to reach the predefined targets and having to adjust for any potential movement of the leg during the pinning process. The potential improvement in terms of accuracy, particularly in the sagittal plane for the tibia, might be explained by the coplanarity of the cut and the ideal adaptation of the jig at the anterior aspect of the tibia. High variations and inaccuracy were observed in the studies reporting the comparison of PSI with the navigation $[8,9]$. The comparison of the prediction intervals observed in our study with those observed with PSI demonstrated a much higher reliability with the robotically assisted TKA [8,9]. A published meta-analysis comparing accuracy of navigation to conventional TKA in 23 published randomized trials provides valuable context for the findings we present here [16]. This study reported findings that 349 of 1160 patients $(30.1 \%)$ operated with conventional instruments showed greater than $2^{\circ}$ of deviation from neutral mechanical alignment in the coronal plane [16]. Their cohort of 1234 navigation-assisted cases included 158 cases (12.8\%) exhibiting greater than $3^{\circ}$ of deviation from the mechanical axis [16].

In conclusion, the results of this in vitro study demonstrate that a high degree of accuracy can be obtained by surgeons using this new robotically assisted TKA system that is based on robotically positioned and stabilized cutting jigs. This study can be considered as the first step in the validation process for this new "cobot" technique and supports use in clinical settings. However, further in vivo studies will be required to investigate first if the accuracy observed in this cadaveric study can be replicated in clinical studies and second to investigate the other potential advantages of this system, such as the time efficiency, optimization of implant positioning, and improvements in patient-reported outcomes.

\section{References}

[1] Halawi MJ, Jongbloed W, Baron S, Savoy L, Williams VJ, Cote MP. Patient dissatisfaction after primary total joint arthroplasty: the patient perspective. J Arthroplasty 2019;34(6):1093-6. https://doi.org/10.1016/j.arth.2019.01.075.

[2] Parratte S, Pagnano MW. Instability after total knee arthroplasty. J Bone Joint Surg Am 2008;90:184-94.

[3] Springer BD, Parratte S, Abdel MP. Measured resection versus gap balancing for total knee arthroplasty. Clin Orthop Relat Res 2014;472:2016-22. https:/ doi.org/10.1007/s11999-014-3524-y.

[4] Parratte S, Pagnano MW, Trousdale RT, Berry DJ. Effect of postoperative mechanical axis alignment on the fifteen-year survival of modern, cemented total knee replacements. J Bone Joint Surg Am 2010;92:2143-9. https://doi.org/ 10.2106/JBJS.I.01398.

[5] Abdel MP, Oussedik S, Parratte S, Lustig S, Haddad FS. Coronal alignment in total knee replacement: historical review, contemporary analysis, and future direction. Bone Joint J 2014;96-B:857-62. https://doi.org/10.1302/0301620X.96B7.33946.

[6] Rivière C, Iranpour F, Auvinet E, Howell S, Vendittoli PA, Cobb J, et al. Alignment options for total knee arthroplasty: a systematic review. Orthop Traumatol Surg Res 2017;103:1047-56. https://doi.org/10.1016/j.otsr.2017.07.010.

[7] Bautista M, Manrique J, Hozack WJ. Robotics in total knee arthroplasty. J Knee Surg 2019;32:600-6. https://doi.org/10.1055/s-0039-1681053.

[8] Scholes C, Sahni V, Lustig S, Parker DA, Coolican MR. Patient-specific instrumentation for total knee arthroplasty does not match the preoperative plan as assessed by intra-operative computer-assisted navigation. Knee Surg Sports Traumatol Arthrosc 2014;22:660-5. https://doi.org/ 10.1007/s00167-013-2670-1.

[9] Lustig S, Scholes CJ, Oussedik S, Coolican MR, Parker DA. Unsatisfactory accuracy with VISIONAIRE patient-specific cutting jigs for total knee arthroplasty J Arthroplasty 2014;29:249-50. https://doi.org/10.1016/j.arth.2013.05.020.

[10] Biant LC, Yeoh K, Walker PM, Bruce WJ, Walsh WR. The accuracy of bone resections made during computer navigated total knee replacement. Do we resect what the computer plans we resect? Knee 2008;15:238-41. https:// doi.org/10.1016/j.knee.2008.01.012.

[11] Bland JM, Altman DG. Statistical methods for assessing agreement between two methods of clinical measurement. Lancet 1986;1:307-10.

[12] Galaud B, Beaufils P, Michaut M, Abadie P, Fallet L, Boisrenoult P. Distal femoral torsion: comparison of $C T$ scan and intra operative navigation measurements during total knee arthroplasty. A report of 70 cases. Rev Chir Orthop Reparatrice Appar Mot 2008;94:573-9. https://doi.org/10.1016/j.rco.2008.03.039.

[13] Casper M, Mitra R, Khare R, Jaramaz B, Hamlin B, McGinley B, et al. Accuracy assessment of a novel image-free handheld robot for Total Knee Arthroplasty in a cadaveric study. Comput Assist Surg (Abingdon) 2018;23:14-20. https:// doi.org/10.1080/24699322.2018.1519038.

[14] Hampp EL, Chughtai M, Scholl LY, Sodhi N, Bhowmik-Stoker M, Jacofsky DJ, et al. Robotic-arm assisted total knee arthroplasty demonstrated greater accuracy and precision to plan compared with manual techniques. J Knee Surg 2018;32:239-50. https://doi.org/10.1055/s-0038-1641729.

[15] Kim TK, Chang CB, Kang YG, Chung BJ, Cho HJ, Seong SC. Execution accuracy of bone resection and implant fixation in computer assisted minimally invasive total knee arthroplasty. Knee 2010;17:23-8. https://doi.org/10.1016/j.knee.2009.06.004.

[16] Hetaimish BM, Khan MM, Simunovic N, Al-Harbi HH, Bhandari M, Zalzal PK. Meta-analysis of navigation vs conventional total knee arthroplasty. J Arthroplasty 2012;27:1177-82. https://doi.org/10.1016/j.arth.2011.12.028. 